\title{
Desigualdades en la provisión de servicios sanitarios
}

Pedro Gallo*

\section{Introducción ${ }^{1}$}

La Constitución española y los Estatutos de Autonomía fueron la base de una nueva organización del Estado en Comunidades Autónomas y el comienzo de un proceso consensuado de descentralización de importantes áreas de gestión pública entre las que se cuenta sanidad. En abril de 1986 la Ley General de Sanidad desarrolló en más profundidad los principios descentralizadores constituciónales en materia de política sanitarià. Creó un Sistema Nacional de Salud similar, en conceptos y objetivos, a los que funcionaban hacía ya tiempo en otros países europeos. Se apostó asi por un modelo caracterizado por la cobertura universal y una financiación mayoritaria con cargo a impuestos, y dotado de elementos de control, de participación ciudadana y de descentralización en la gestión y la organización de los servicios sanitarios.

El proceso de descentralización ha sido descrito suficientemente en otras contribuciones y publicaciones (De MIGUEL, 1979 y 1985, RONDINELLI, 1981, ARTIGAS, 1990, BENNET, 1990 y 1992, Milis et al., 1990, De Miguel y Rodríguez, 1990, Elola, 1991, Generalitat de Catalunya, 1992, NaVarRo, 1992, RoEmer, 1993, Rico, 1997 y 1998). Se argumenta a su favor en base a: (i) los beneficios potenciales de una mayor racionalidad en la organización de los servicios en áreas geográficas más pequeñas, (ii) una mayor participación de las distintas Comunidades Autónomas en la administración de sus propios servicios sanitarios, lo que lleva a la elaboración de planes de salud más acordes con los problemas y necesidades sanitarias locales, (iii) un mayor y más exhaustivo control de los costes y duplicidades de servicios, (iv) la reducción de las desigualdades y los desequilibrios sanitarios, (v) la mejor integración de actividades sanitarias públicas y privadas y, (vi) en general, la reducción del control central sobre programas que son competencia de administraciones sanitarias subestatales (Milus et al., 1990).

Atender mejor las necesidades de la población y corregir las desigualdades son dos de los principales apoyos de la descentralización sanitaria. Ciertamente, la asignación de recursos en base a un conocimiento más exhaustivo de las necesidades en salud y en servicios sanitarios es más factible si las unidades de gestión de estos recursos están más próximas a los problemas sanitarios. Sin menospreciar las dificultades derivadas de la asunción de nuevas responsabilidades y áreas de gestión $\left(\mathrm{G}_{\mathrm{A}}\right.$ LLO, 1994), los problemas de salud que afrontan las Comunidades con competencias transferidas no son muy diferentes de los que afectan al país en su conjunto. Sin embargo, el margen de gestión fruto de la descentralización permitirá que estas autoridades sanitarias puedan actuar de manera más autónoma, flexible y localista ante estos problemas. Conocer mejor la distribución de la salud y de la mala salud, de los servicios disponibles así como la estructura y organización de estos servicios se convierte en el eje fundamental de la gestión sanitaria a nivel de cada Comunidad Autónoma.

La presencia de desigualdades en salud y servicios sanitarios son cuestiones que afectan a todos los organismos públicos de gestión sanitaria. La equidad es un principio reconocido constitucionalmente (arts. 31.2, 41, 43.1, 49), en disposiciones legales como la Ley General de Sanidad (arts. 3.2, 3.3, 6.4, 12, 16, 46), así como en los respectivos Estatutos de Autonomía y en sus posteriores desarrollos legislativos. Bajo estos preceptos, la corrección de las desigualdades debería constituir uno de los objetivos básicos de la actuación de los organismos públicos 
nacionales y autonómicos. Sin embargo, aunque el objetivo de la equidad es omnipresente en estos textos, existe una vaguedad importante en cuanto a su definición, y un vacío considerable en cuanto a cómo se deben medir las desigualdades en salud y en servicios sanitarios.

La equidad es un concepto con grandes connotaciones ideológicas. Las distintas escuelas de pensamiento abordan su definición desde puntos de vista muy diferentes, incluso radicalmente opuestos. El utilitarismo, el neoliberalismo, el pensamiento rawlsiano, el igualitarismo o la teoría de las capabilidades del reciente premio Nobel Sen, son algunas de estas aproximaciones al término equidad (LE GRAND, 1976, RAWLS, et al., 1988, Culyer, 1991, Culyer et al., 1993, Pereira 1993, PuYOL, 1997). No obstante, es posiblemente el igualitarismo el enfoque más claramente presente en el desarrollo y funcionamiento de la mayoría de los sistemas sanitarios occidentales (SUGDEN, 1983, VAN DoORSLAer et al., 1993). El igualitarismo apuesta por un concepto de equidad según el cual los servicios sanitarios deben proveerse de acuerdo con las necesidades de la población y financiarse en consonancia con la capacidad de pago de los individuos.

Tomando esta aproximación a la equidad, y dentro del marco de la descentralización apuntado, el papel de las autoridades sanitarias a nivel autonómico se concreta en cumplir con una provisión de servicios sanitarios que esté de acuerdo con las necesidades de los individuos, y asimismo en elaborar las políticas sanitarias necesarias para la corrección de las desigualdades existentes y emergentes en su ámbito de actuación. El estudio de estas desigualdades a nivel de cada Comunidad Autónoma será un instrumento que informará el diseño de los mecanismos de corrección más efectivos.

\section{El estudio de las desigualdades en España}

La investigación sobre desigualdades en sanidad ha estado muy condicionada tanto por el sujeto de estudio como por la forma en que se ha aproximado su medición. Se habla de manera diferente de desigualdades en salud y de desigualdades en servicios sanitarios, de necesidades de salud y de necesidades de servicios sanitarios, de fines y de medios. Estas distinciones sugieren, entre otras cosas, que hay cuestiones como la vivien$\mathrm{da}$, la educación, las infraestructuras, los ingresos, o los estilos de vida, que tienen importantes implicaciones en el nivel de salud de la población. Los servicios sanitarios no son, pues, los únicos determinantes de la salud de los individuos (DHSS, 1980; Benzeval et al., 1995; PerCi-Smith et al., 1995; Gallo,
1998). Ello tiene una especial importancia por cuanto define el papel de las autoridades sanitarias a nivel nacional o autonómico, así como el de sus respectivas políticas, como responsables de sólo un ámbito de los muchos que pueden afectar la salud de la comunidad: atender las necesidades y corregir las desigualdades en servicios sanitarios en el ámbito geográfico de competencia.

En España el estudio sobre desigualdades en sanidad tiene una gran tradición y cuenta con una excelente aceptación en las revistas especializadas. BENACH identifica en un análisis bibliométrico más de doscientos treinta estudios específicos sobre desigualdades en salud y desigualdades en servicios sanitarios publicados durante el período 1978-1994 (BENaCH, 1995). Sin duda se trata de un producción científica fructífera, que no sólo tiene como referencia al país en su conjunto sino ciertas Comunidades Autónomas y ciudades de España ${ }^{2}$.

Dos cuestiones merecen destacarse de estas aportaciones. En primer lugar, se trata en gran parte de estudios que analizan las desigualdades relacionadas con la mortalidad. Ello es sin duda relevante por cuanto ha generado un conocimiento vasto sobre cómo muere la población, qué enfermedades causan la muerte y cómo se distribuye la mortalidad según edades, géneros y variables socioeconómicas como clase social, nivel de estudios o ingresos.

Sin embargo, el interés del investigador y del gestor de servicios sanitarios no debe centrarse exclusivamente en cómo y por qué muere la población sino especialmente en cómo vive $y$, por extensión, en cómo utiliza los servicios sanitarios durante ese período de vida. No es posible responder a estas preguntas con los datos recogidos por las estadísticas de mortalidad o con los resultados ofrecidos por los estudios sobre desigualdades en mortalidad. Para estudiar desigualdades en servicios sanitarios es necesario entender cómo se proveen estos servicios a los diferentes grupos socioeconómicos, más allá del concepto de mortalidad, ya que existen multitud de enfermedades que no son letales y que implican en conjunto un gran consumo de servicios y de recursos de todo tipo.

En segundo lugar, cabe destacar la importancia de las fuentes de datos de las que se nutren estos estudios. Son mayormente las mencionadas estadísticas de mortalidad, las estadísticas de morbilidad hospitalaria, los registros de pacientes y las encuestas de salud. Estas últimas se han constituido en uno de los instrumentos más apreciados para el estudio de desigualdades en salud y servicios sanitarios. La evolución de la producción científica se ha visto influenciada por la aparición y uso de estas fuentes de datos. Así, por ejemplo, la no existencia de encuestas de salud a nivel autonómico hasta hace pocos años ha condicionado enormemente el estudio de las desigualdades en salud y servicios sanitarios a ese nivel de actuación política. 
La tendencia en la publicación de estudios sobre desigualdades observada por BENACH y otros autores se ha mantenido a lo largo de los últimos años. Se pueden identificar así estudios a los tres niveles de definición de las políticas, nacional, regional y local, tratando entre otros aspectos sobre mortalidad (ARIAS y BORrEll, 1998, Segura y FernánDEZ, 1998, LloRCA et al., 1998), morbilidad en condiciones clínicas concretas (LaTOUR-PÉREZ et al., 1995; Doz et al., 1996; LUENGO et al., 1996, ROHLFS et al., 1998), salud materno-infantil (DíEZ et al., 1995, NAVARRO-RUBIO et al., 1995, SÁnChEZ-FERnÁNDEZ et al., 1995, VAiERo et al., 1995), servicios sanitarios específicos (IBÁNez-FraILE et al., 1995, RuIz et al., 1995, FERNÁNDEZ DE LA HOZ y LEÓN, 1996), y discapacidades (REGIDOR et al., 1997).

Las conclusiones más relevantes de esta enorme producción científica confirman la importancia de las variables género, edad, ámbito rural-urbano, regiones y grupos socioeconómicos en la distribución de la salud y en el uso de servicios sanitarios específicos. No obstante, pocos son los estudios sobre desigualdades que exploran la relación entre el grado de utilización de servicios sanitarios y las necesidades sanitarias de la población. Ciertamente, se conocen cada vez mejor las relaciones entre variables como edad, género, grupos socioeconómicos y salud, por las que se ha podido constatar un gradiente socioeconómico en esta relación. Los individuos de clase social más baja, de más bajos ingresos o de peores niveles educativos dicen tener un peor estado de salud, midiendò este último con una gran variedad de indicadores. Sin embargo, se conoce poco sobre si la provisión de servicios sanitarios se corresponde con ese gradiente socioeconómico en la distribución de la salud o la mala salud. Es decir, si las autoridades sanitarias proveen servicios sanitarios de acuerdo con criterios de necesidad, y así aquellos que están en peor estado de salud reciben servicios sanitarios en proporción a esos niveles de mala salud ${ }^{3}$.

¿Qué evidencia existe de que el Sistema Nacional de Salud, o los servicios de salud de las Comunidades Autónomas, proveen servicios sanitarios según las necesidades de la población? La respuesta a esta pregunta suscita otras preguntas previas como qué entendemos por necesidad, o qué instrumentos de medida del grado de equidad se ajustan a este principio igualitarista.

\section{La medición de las desigualdades en servicios sanitarios}

La medición de las desigualdades en servicios sanitarios no está exenta de dificultades. La primera limitación la supone la disponibilidad, fiabilidad y accesibilidad de las fuentes de datos necesarias en el ámbito geográfico que se quiere estudiar. La segunda de ellas hace referencia al uso de instrumentos de medida adecuados, sean éstos rangos, índices específicos o curvas de concentración.

Las bases de datos necesarias para el estudio de las desigualdades en servicios sanitarios se alejan de las estadísticas y registros de mortalidad, comunes en otros estudios sobre desigualdades, para dirigirse a las encuestas de salud como fuentes de información. El desarrollo de estas últimas ha espoleado la aparición de estudios sobre desigualdades. Las encuestas nacionales de salud, las encuestas de salud de Barcelona, o las más recientes encuestas de salud autonómicas (ESCA, 1994) se han destacado como piezas fundamentales para el estudio de las desigualdades en servicios sanitarios. Si la definición de políticas de corrección de desigualdades se realiza cada vez más en referencia al contexto descentralizado de la política sanitaria, el desarrollo de bases de datos del tipo encuestas de salud a nivel de Comunidad Autónoma deja de ser un capricho de los investigadores y se convierte en un instrumento esencial de política y gestión sanitaria.

En base a los datos ofrecidos por las encuestas de salud se plantea la medición de las desigualdades en servicios sanitarios. De acuerdo con la definición igualitarista de equidad, se trata de estudiar en qué medida la provisión de servicios persigue criterios de necesidad, proveyendo igual atención sanitaria ante igual necesidad de esa atención sanitaria. El gestor y el investigador se enfrenta, a una ecuación con dos elementos, necesidad y atención sanitaria, en un marco de prestación de servicios descentralizado.

Precisar qué son necesidades es, y continuará siendo, centro de un debate intelectual intenso abordado desde múltiples puntos de vista. La construcción de indicadores de necesidad ha ocupado la mente del sociólogo, del economista, del médico y del filósofo durante años. Sin embargo, la necesidad de hacer operativo este término en referencia a salud y sanidad ha inclinado la balanza a favor de conceptos como el de morbilidad, la presencia de enfermedad o riesgo de enfermedad en la población, así como las medidas subjetivas de salud. Los indicadores más comúnmente utilizados han sido la autopercepción de salud, la presencia de enfermedades crónicas y agudas y los factores limitantes de estas condiciones físicas y psicológicas mermadas ${ }^{4}$. La atención sanitaria ha sido un concepto más fácil de concretar. La gran mayoría de los estudios sobre desigualdades en servicios sanitarios publicados nacional e internacionalmente definen atención sanitaria como los servicios recibidos por la población en los distintos niveles asistenciales, es decir, atención primaria, de especialista y hospitalaria.

Un instrumento metodológico adecuado para la medición de desigualdades en servicios sanitarios, que permita estimar el 
grado en el que la atención sanitaria se provee siguiendo criterios de necesidad, debe cumplir con tres condiciones importantes. En primer lugar, se requiere que sea capaz de reflejar la dimensión socioeconómica de las desigualdades en salud y servicios sanitarios identificado en los estudios sobre desigualdades. En segundo lugar, se precisa que la experiencia en salud y consumo de servicios sanitarios de toda la población quede reflejada. Por último, dicho instrumento debe ser sensible a cambios en la distribución de la población según ingresos, clases sociales o niveles educativos. De acuerdo con estos tres principios básicos, el instrumento mejor considerado para la medición de estas desigualdades son las curvas de concentración de necesidad y atención sanitaria, así como los índices que de ellas se construyan 5 .

Las curvas de concentración representan gráficamente las distribuciones acumuladas de enfermedad y atención sanitaria en un diagrama de tipo caja. La población se ordena de mayor a menor ingresos, por ejemplo, en el eje de abscisas. La distribución de la enfermedad se representa en el eje de ordenadas. Así, las curvas de concentración reflejarán cómo se distribuyen la enfermedad y la atención sanitaria de acuerdo con la población ordenada según una variable socioeconómica de interés. Una distribución perfectamente proporcional coincidirá con la diagonal del cuadro. Una distribución desigual se apartará de la diagonal dibujando curvas por encima o por debajo de esa diagonal. Los índices se calcularán en función de las diferencias entre la diagonal y las curvas de concentración dibujadas, como se muestra en el gráfico 1. En el eje horizontal se representan porcentajes acumulados de población ordenados según ingresos. En el eje vertical se muestran los porcentajes acumulados de enfermedad o necesidad. La encuesta de salud permite obtener la información necesaria para construir las distintas curvas. La curva superior (A) refleja cómo se distribuye en la población la enfermedad crónica funcionalmente limitante, por ejemplo. Esta distribución es resultado de preguntas concretas al respecto en la encuesta de salud y de cómo los individuos en los distintos grupos de ingresos responden a dichas preguntas. Así, figuradamente, del total de individuos que respondieron afirmativamente, la curva (A) apunta que el $30 \%$ se encuentra en el grupo a1. de ingresos más bajos, el 25 por ciento en el siguiente grupo de ingresos, y así hasta completar la distribución porcentual. Una curva como la (A), por encima de la diagonal, identifica una distribución de la enfermedad crónica según la cual a medida que aumentan los ingresos disminuye la presencia de dichas enfermedades. La diferencia con la diagonal muestra el grado de desigualdad en la distribución de estas enfermedades crónicas.

De igual manera, la buena salud también puede ser representada gráficamente mediante curvas de concentración. Así, la curva (B) por debajo de la diagonal identifica, también figuradamente, la distribución de otro indicador de necesidad, la buena salud en general, obtenida también de las encuestas de salud. En este caso la buena salud se distribuye de forma que se concentra en los grupos de ingresos superiores, confirmando la existencia de un gradiente socioeconómico en cómo se distribuye la salud de la población: las desigualdades favorecen a los grupos de ingresos más altos. Otros indicadores similares de salud y de enfermedad pueden ser construidos haciendo uso de la información recogida por las encuestas de salud.

De esta forma se conocerá cómo se distribuye en la población la enfermedad o la necesidad, es decir, la primera parte de la ecuación. Faltará conocer cómo se distribuye la atención sanitaria ante esas desigualdades. Para ello también se hace uso de curvas de concentración. Éstas muestran gráficamente la atención sanitaria recibida por los distintos grupos de ingresos. Las encuestas de salud recogen en está información el número de consultas de atención primaria, especialista y estancias hospitalarias realizadas por los distintos grupos, y se traduce todo ello a una unidad monetaria común, de fácil representación por medio de una única curva de concentración de gasto según ingresos. La curva (C), por encima de la diagonal, muestra una distribución hipotética de gasto en relación a la curva (A).

Una vez representadas las curvas de concentración de necesidad (A), por ejemplo, y atención sanitaria (C), se procede al cálculo de los índices de desigualdad. Si las dos curvas coinciden, es decir, presentan iguales distribuciones de necesidad y atención sanitaria, se estará ante una situación de equidad bajo la cual los que están en igual necesidad sanitaria reciben igual proporción de gasto. La diferencias entre las curvas denotarán situaciones de desigualdad en el uso de servicios sanitarios por las que los grupos de bajos ingresos, por ejemplo, estarán recibiendo una proporción inferior $(A>C)$ o superior $(A<C)$ de gasto a la de su proporción de necesidad. Los índices de Le Grand representan numéricamente estas diferencias.

Todos los estudios realizados en países de nuestro entorno que utilizan este tipo de indicadores llegan a la conclusión de que la salud se distribuye de forma no igualitaria, favoreciendo a las clases más pudientes o de mayores ingresos, lo que también muestran los estudios sobre desigualdades en salud en nuestro propio contexto. Pero, como se ha mencionado, en el estudio de las desigualdades en servicios sanitarios no sólo interesa conocer cómo se distribuye la salud o la mala salud, sino particularmente cómo se distribuye la atención sanitaria ante esa distribución de salud o mala salud. Pero, ante ello... ¿qué evidencia empírica encontramos en la literatura de este segundo tipo de estudios? 


\section{Giréfico 1}

\section{Curvas de Concentración}

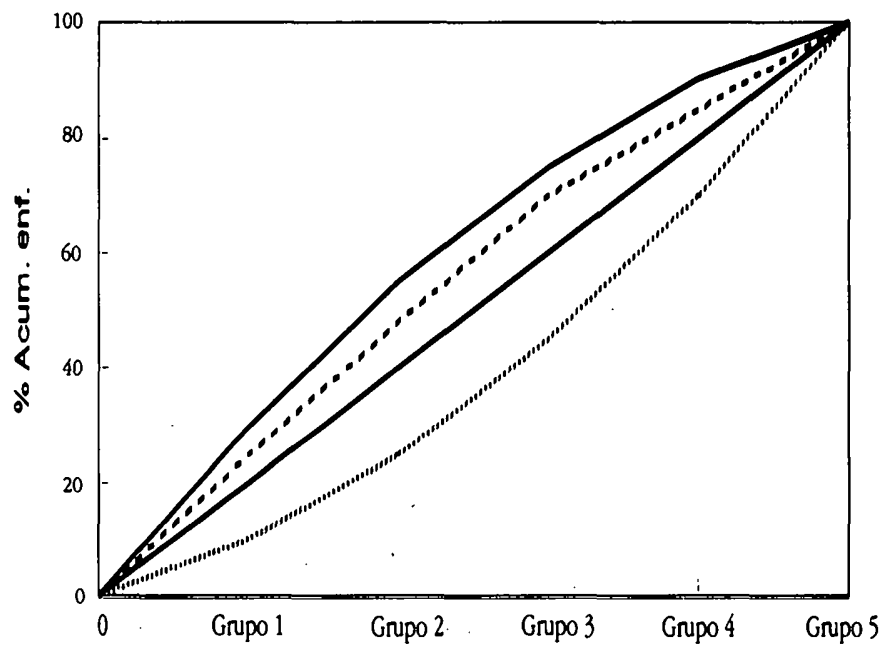

\% Acum. población

\begin{tabular}{c} 
Leyenda \\
$-45^{\circ}+{ }^{(A)}-$ (B) $\quad \cdots .$. (C) \\
\hline
\end{tabular}

\section{Una perspectiva internacional}

VAN DOORSLAER et al. (1993) ponen de manifiesto los trazos generales de las desigualdades en servicios sanitarios en más de 15 países de Europa y América haciendo uso de esta metodología. Este estudio incluye el caso de España entre los países comparados, lo que además facilita la comparación de ciertos aspectos de la provisión de servicios sanitarios de nuestro país. Los distintos autores para cada país estudiado utilizan bases de datos similares a la Encuesta Nacional de Salud utilizada en el caso de España, y miden el grado de equidad de los distintos sistemas sanitarios en base a curvas de concentración como las expuestas.

El gráfico 2 recoge los resultados principales de estos estudios mediante los indices de equidad de LE GRAND, no estandarizados por edad y género ${ }^{6}$, para una variedad de indicadores de necesidad (mala salud, enfermedad crónica limitante, enfer- medad crónica y enfermedad aguda). Estos índices muestran las diferencias entre curvas de concentración de necesidad y gasto asignado a la población según grupos de ingresos. Se calcularon tantos índices como indicadores de salud o mala salud se utilizaron, todos ellos extraídos de preguntas específicas en las encuestas de salud de los distintos países.

El rango teórico en el que se mueve el índice LE GRAND es $[-1,+1]$ por lo que se puede constatar que las desigualdades mostradas no son muy grandes en términos globales, y serían aún menores después de ser estandarizadas por edad y género. En cualquier caso, el gráfico muestra cómo todos los sistemas sanitarios representados presentan un cierto grado de desigualdad que los aparta de la proporcionalidad, identificada con el valor cero. Estas desigualdades convierten a los sistemas sanitarios en progresivos (cuando el índice es inferior a cero) o regresivos (superior a cero) en lo que a la relación entre servicios sanitarios y necesidad se refiere. El gráfico 2 también refleja que el uso de uno u otro indicador de necesidad da lugar a resultados de diferente signo, excepto en los casos de EE.UU., Reino Unido y España. En estos tres casos los índices no estandarizados se muestran siempre regresivos en cuanto a la provisión de 
servicios sanitarios, independientemente del indicador de necesidad que se haya utilizado.

Sin embargo, el argumento central de este texto consiste en defender que, para España, este tipo de estudios son, si cabe, de más relevancia en contextos geográficos más concretos y descentralizados. Si de todo estudio sobre objetivos de política sanitaria deben extraerse implicaciones de política sanitaria y éstas se definen a nivel autonómico en el caso español, entonces conviene pensar que es a ese nivel de actuación al que se ha de dirigir el análisis.

La realización de la Encuesta de Salud de Cataluña (ESCA, 1994) ha permitido llevar a cabo este tipo de análisis para el caso de Catalunya, Comunidad Autónoma con competencias transferidas en materia de sanidad desde principios de los ochenta. Haciendo uso de esta misma metodología y en base a los datos aportados por la ESCA se ha podido medir el grado de equidad del sistema sanitario catalán, ayudando a definir y diseñar políticas más acordes a ese contexto sanitario.

Tal y como refleja el gráfico 2, el caso de Cataluña se asemeja más al de otros países europeos que al propio de España. Tanto el grado de equidad del sistema como el sentido de esa desigualdad, progresivo o regresivo según indicadores, presentan más semejanzas con Holanda o Dinamarca que con el conjunto del Estado español. De hecho, el caso catalán encaja bien en el grupo de aquellos otros países para los que el uso de un indicador de salud u otro es de especial importancia para la medición de las desigualdades en servicios sanitarios a nivel global ${ }^{7}$.

Si bien los resultados presentados en el gráfico 2 son globales y por tanto se refieren a los sistemas de salud en su conjunto, es preciso destacar la necesidad de estudiar en mayor profundidad la influencia de variables específicas como género y edad, medio rural o urbano u otras variables socioeconómicas de interés para concretar las causas o factores que explican las desigualdades.

La universalización de la cobertura sanitaria a prácticamente toda la población española ha sido un argumento comúnmente utilizado para explicar la equidad en los sistemas sanitarios (DE MIGUEL, 1994). Otras características como la extensión de la atención primaria, el incremento de los recursos en el sistema, particularmente en lo que se refiere a hospitales, o el proceso de descentralización han sido también citadas con cierta frecuencia (REGIDOR et al., 1996; GuTtÉRREZ, 1997). En este sentido, se ha mostrado aquí que un ámbito de gestión sanitaria descentralizado como en Cataluña podría asociarse a unos mayores niveles de equidad. No obstante, el

Gráfico 2

Índices de desigualdad de Le Grand

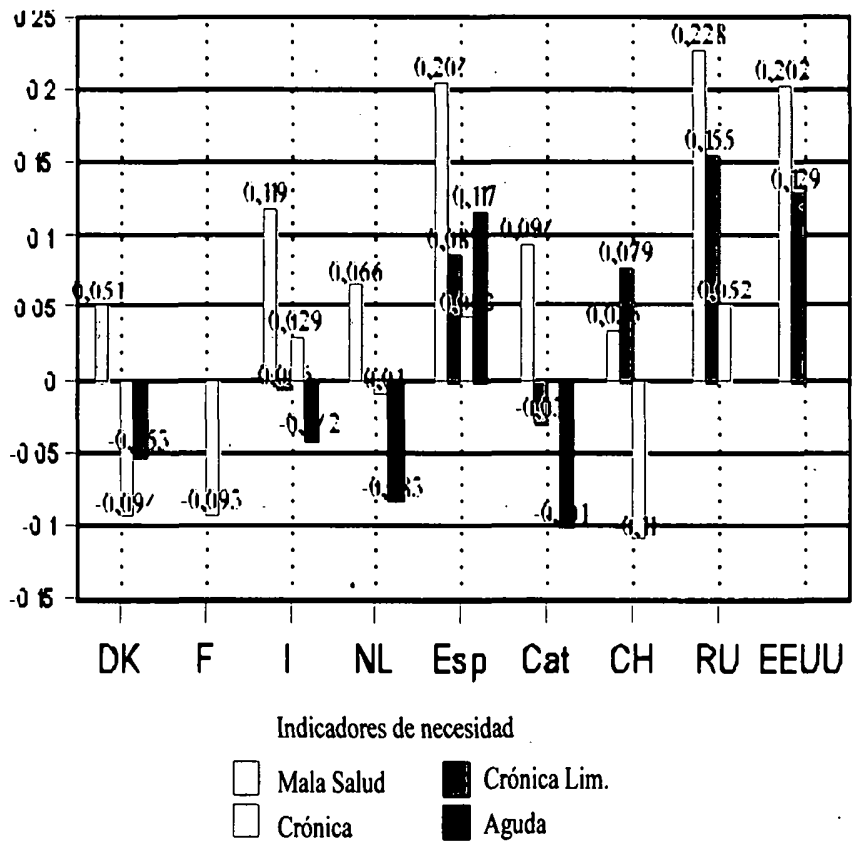


estudio de sólo un caso, así como la naturaleza de las fuentes de datos utilizadas no permiten en modo alguno establecer relaciones causales. Según los indicadores estudiados, Cataluña muestra un grado de equidad diferente que España en cuanto a la provisión de servicios sanitarios. Este resultado sugiere que hay otros factores, más allá de la cobertura universal, que tienen efectos sobre la equidad del sistema.

Tal como sugiere LE Grand (1982), las desigualdades en servicios sanitarios pueden explicarse mejor estudiando las tasas de utilización de servicios sanitarios de los que están en mala o buena salud, que atendiendo a la presencia de enfermedades agudas o crónicas entre los grupos socioeconómicos. Las tasas de utilización de servicios sanitarios constituyen sin embargo una de las variables menos estudiadas entre los investigadores. En la literatura se apuntan, no obstante, una serie de factores que se creen importantes a la hora de explicar estas tasas de utilización. Entre ellos cabe destacar de nuevo el género, la edad, los ingresos económicos u otras variables que, en conjunto, determinan diferentes percepciones sobre la salud, sobre los síntomas o las urgencias, el acceso a servicios sanitarios concretos, las diferentes capacidades de influir en el médico de primaria para una rápida derivación a otros niveles asistenciales, un distinto conocimiento de cómo funcionan los sistemas sanitarios, una desigual capacidad de afrontar una comunicación efectiva con los profesionales de la salud, o de tratar con los aspectos más administrativos y burocráticos que imponen los sistemas sanitarios.

\section{Implicaciones de política sanitaria}

El estudio de las desigualdades en salud y servicios sanitarios debe tener como objetivo último informar las políticas sanitarias a este respecto. Sin dejar de estudiar la evolución de las desigualdades, se deben construir las bases necesarias para el adecuado diseño y evaluación de las políticas de corrección de las desigualdades. Ésta será una pieza clave del debate sanitario en el futuro, particularmente por lo que se refiere a los medios, los instrumentos, y los niveles de actuación, de coordinación y de participación más efectivos.

Las primeras implicaciones de política sanitaria deberían centrarse en las variables que afectan la desigual distribución de la salud o la mala salud en la población. La políticas sanitarias deben insistir sobre las medidas preventivas, la educación para la salud, la salud pública en general, el estudio de las variables epidemiológicas de incidencia, prevalencia y riesgo de las enfermedades en el contexto de actuación, así como la asistencia sanitaria adecuada. No obstante, se entiende que hay otros muchos factores que determinan la salud de los individuos y que escapan a la actuación directa de la política sanitaria y que son igualmente importantes como materia de estudio en cuanto a la magnitud y efectividad de sus intervenciones.

En segundo lugar, cabe dirigir el gasto sanitario hacia los grupos más desfavorecidos no por ello olvidando el cariz universalista de la cobertura en la prestación de servicios sanitarios. Igualmente, debe destacarse la necesidad de estudiar en mayor detalle la existencia de patrones de utilización diferentes según grupos socioeconómicos, género y edad. En este sentido, el estudio de las desigualdades a nivel global puede esconder, y por tanto no reflejar, diferentes patrones de utilización de servicios sanitarios en consonancia con los diferentes niveles asistenciales de atención primaria, especialista y atención hospitalaria (Gallo et al., en fase de edición).

Si las razones por las que existen desigualdades en servicios sanitarios son la presencia de diferentes tasas de utilización según grupos socioeconómicos, edad o género, se debe entonces estudiar por qué se dan dichas diferencias, y diseñar políticas e incentivos acordes que permitan cambios de actitudes, percepciones y expectativas en la población respecto al sistema sanitario.

Los esfuerzos de investigación en el futuro deben igualmente dar continuidad a los aspectos metodológicos de medición de las desigualdades. El estudio de las desigualdades se ve afectado por los instrumentos de medida y por las bases de datos disponibles, aspectos ante los cuales la investigación en servicios sanitarios y la aplicación de las nuevas tecnologías de la información pueden ofrecer importantes avances. Por último, el estudio de las desigualdades no debe desligarse del estudio del impacto de las medidas concretas de política sanitaria, es decir, de lo que hay evidencia de que funciona y de lo que no es eficaz en la corrección de desigualdades en contextos similares al propio. Ello debe fomentarse a los tres niveles de gestión y política sanitaria, micro, meso y macro, es decir, desde la práctica clínica más cercana al individuo a las trascendentes decisiones de gestión estratégica y de reforma sanitaria.

\section{Conclusión}

Teniendo en cuenta los procesos de descentralización sanitaria, la investigación sobre las desigualdades en salud y servicios sanitarios debe enfocarse más adecuadamente al nivel de actuación política. Las Comunidades Autónomas deben asumir el diseño de las políticas de corrección de desigualdades en servicios sanitarios, así como fomentar la investigación y evaluación sobre la efectividad de dichas políticas. 
El camino abierto es ciertamente un camino inexplorado ante el que las autoridades sanitarias deben ejercer un rol central, no sólo en el cumplimiento del objetivo de equidad en la prestación de servicios, sino también en la orientación del resto de políticas que tienen un impacto en la salud de la población.
- Agència d'Avaluació de Tecnologia Mèdica, Servei Català de la Salut. Departamento de Sociología y Análisis de las Organizaciones, Universitat de Barcelona.

' El autor agradece el apoyo de The Richard TTTMUSS-MEINHARD Foundation, el Institut de Ciències Politiques i Socials (ICPS), y la Fundació Jaume Bofill. Quiere reconocer igualmente los estímulos intelectuales de Jesús $M$. de Miguel, así como del resto de compañeros del Departamento de Sociologia y Análisis de Organizaciones de la Universidad de Barcelona. Durante los últimos cuatro años ha recibido el apoyo en estos temas de investigación de Albert J. Jovell, Mateu Serra-Prat y Alícia Granados de l'Agència d'Avaluació de Tecnologia Mèdica. En la London School of Economics quiere agradecer los comentarios y sabias sugerencias de Julian Le Grand, Steen Mangen y Howard Glennerster. Y last but by no means least, el autor desea hacer constar su agradecimiento a Patrícia García-Durán por su constante apoyo y espiritu positivo.

- Los estudios pueden agruparse en seis categorías atendiendo también al nivel de actuación de política:

(i) Mortalidad en el ámbito nacional, (ii) Mortalidad en los ámbitos regional y local, (iii) Morbilidad y estilos de vida a nivel nacional, (iv) Mobilidad y estilos de vida a niveles regional y local, (v) Utilización y acceso a servicios sanitarios a nivel nacional, (vi) Utilización y acceso a servicios sanitarios a niveles regional y local. Pam una relación completa de los estudios por autores y temas ver BENACH (1995) así como NavA. RRO Y BENACH (1996a, 1996b).

"Entre los primeros intentos de medir el grado de equidad así entendido se encuentra la aportación de BanDRés sobre la progresividad del gasto público en España (BANDRÉs, 1991), aunque no centrado en servicios sanitarios. En servicios sanitarios cabe destacar las contribuciones de RodRfGUez et al., (1993), REGidor et al., (1996) y ABÁSOLO et al., (1998).
' Los conceptos de necesidad, atención sanitaria y equidad se han abordado exhaustivamente. A este respecto cabe senalar las contribuciones de AliACH et al.,,, (1987), ANDERSON y NEWMAN (1973), ARBLASTER et al.,, (1996), ANTONOVSKY (1989), BAUMOL (1986), Benzeval et al., (1995), BlaCK (1991), DHSS (1980), BirTCH et al., (1993), BlaNE (1995), BlaXTER (1989, 1997), Blazer et al., (1995), Borrás (1994), BrAYles et al., (1983), Cavalaars et al, (1998), Counss \& KLein (1980), Culyer (1988, 1993), Dworkin (1981), Fox (1989), Gerdtham (1997), Green (1988), Hasav (1989), House et al., (1990), ILLLLEY and SVENSSON (1986), JudGe (1998), Kuller (1995), LAIRSON et al, (1995), LE GRAND (1978, 1982, 1987a, 1991), LeE et al., (1995), LeMKOw (1980) MACINTYRE (1997), MACKENBACH y KUNST (1997), MANOR et al., (1997), MARMOT et al., (1997), De Miguel (1986), MoOney (1987), Monroe (1992), Mossey et al., (1982), O'. DONNELl y Propper (1991a,1991b), POLAND et al., (1998), PUfFer (1985), ROSENBERG y HANLON (1996), SCRIVENER et al., (1986), SIEGRIST (1995), SHI et al., (1997), VAGERO (1991), VAN DOORSLAER et al., (1993), VAN DER MEER et al., (1996), WADSWORTH (1997), WAGSTAFF et al., (1991a, 1991b, 1991c, 1994), WHITEHEAD (1992), WHTELEGG (1982), YERGAN et al., (1981) y ZIERLER (1996).

" Para una lectura y estudio completo de los distintos instrumentos de medición de las desigualdades en salud y servicios sanitarios ver WAGSTAFF et al., (1991c), donde se resumen la utilidad, potencial y limitaciones de los Rangos, Coeficientes de Gini, Pseudo-coeficientes de Gini, Índices de Disimilaridad, Índices Relativos, y Curvas e Índices de Concentración.

"El estudio no hace uso de los mismos indices estandarizados. No obstante, hay evidencia suficiente como para suponer que el proceso de estandarización tiene un efecto decreciente sobre la magnitud de las desigualdades recogidas aquí.

' Para un detalle exhaustivo del caso de Cataluña ver Gallo et al., (en fase de edjción).

\section{Bibliografia}

ABÁSOLO, I., JONES, A. y MANNING, R. Equidad en la provisión y acceso al médico general priblico en España. XVIII Jomadas de Economía de la Salut (Vitória-Gasteiz),1998.

ALONso, J. y ANTO, J.M. .Desigualdades de salud en Barcelona. Gac Sanit 1988;2(4):412.

AliACH, P., CARR-HIL, S. y lLLSEY, R. Les Inéqualités Sociales en santé en France et Grande-Bretagne. DF-Iserm, Paris 1987.

ANDERSON, R. y NEWMAN, J.F. Societal and individual determinants of medical care utilisation in the United States. Milbank Mem Fund Q, 1973;51:95-124.

ANTONOvSKY, A. Social inequalities in health: a complementary perspective. En Fox J. Ed. Health Inequalities in European countries, Gower, 1989.

ARBER, S. Gender and class inequalities in health: understanding the differentials. En Fox, J. Ed. Health Inequalities in European countries, Gower, 1989.

ARIAS, L.C. y BoRREu, C. -Mortality inequalities according to education in the city of Barcelona. Med Clin 1995;110(5):161-6.

ARTIGAS, J. Healib services decentralization in Spain. En MiLLS, A. et al., Health system decentralisation. Concepts, issues and country experience, WHO, 1990.
BandRES, E. Progresividad, equidad y efectos distributivos de los gastos sociales en España. Hacienda Püblica Española, 1991;117:17-42.

BaumoL, WJ. Superfairness: Applications and theory. The MIT Press, 1986.

BeNACH, J. •Análisis bibliométrico de la desigualdades en salud en España (19801994). Gaceta Sanitaria, 1995;9:251-264.

BENNETT, R.J. Decentralisation, local governments and markets:Towards a post-welfare agenda. Oxford, Clarendon Press, 1990.

BENZEVAL, M., JUDGE, K. y WhTTEHEAD, M. Tackling inequalities in bealth: an agenda for action. Kings Fund Institute, London, 1995.

BiRCH, S., EYLES, J. y NEWBOLD, K.B. .Esquitable access to health care: methodological extensions to the analysis of physician utilisation in Canada. Health economics 1993;2:87-101.

BLACK, D. Inequalities in Health. Public Health 1991,105:23-27.

BlanE, D. .Editorial: Social Determinants of Health - Socioeconomic Stanus, Social Class, and Ethnicity. Am J Public Health 1995;85(7):903-904. 
BIAXTER, M. .Whose fault is it? People's own conceptions of the reasons for health inequalities. Soc Sci Med 1997, 44(6)747-756.

BLAXTER, M. AA comparison of measures of inequality in morbidity. En FOX, J. Ed. Health Inequalities in European countries, Gower, 1989.

Blazer, D.G., Landerman, L.R., Flllevinaum, G. y Horner, R. Health Services Access and Use among Older Adults in North Carolina: Urban vs Rural Residents. Am J Public Health. 1995;85(10): 1384-1390.

Borrajo, E. .Comunidades Autonomas y seguridad social, Papeles de Economía Espaniola $1982 ; 12 \cdot 13: 187-196$.

BorRর́, J.M. •La utilització dels serveis sanitaris. Gac Sanit 1994;8:30-49.

BORRELL, C. y ARIAS, A. Socioeconomic factors and mortality in urban settings: the case of Barcelona. J Epidemiol Community Healtb 1995;49:460-465.

BroYles, R.W., Manga, P., Binder, D., et al., the use of physician services under a National Health Insurance Scheme: an examination of the Canadian health survey. Med Care 1983;21:1037-54.

CAls, J., CAsthlia, E.J. y De Miguel, J.M. .Desigualdad y morbilidad. I Simposio sobre Igualdad y Distribución de la Renta y la Riqueza. Vol VIII, Fundación Argentaria, Madrid, 1991.

Cavelaars, A.E., Kunst, A.E., Geurts, J.J., Crialesi, R., Grotvedt, L., HelmerT, U., LA. helma, E., lundberg, O., Matheson, J., MielK, A., Mizrahi, A., Rasmussen, N.K., ReglDOR, E., SPUNHLER, T., MACKENBACH, J.P. .Differences in self-reported morbidity by educational level: a comparison of 11 western European countries. $J$ of Epidemiol Community Health 1998;52(4):219-27.

COLlado, A. y DE MIGUELl, J.M. .Desigualdades sanitarias y sociales: análisis de la Encuesta Nacional de Salud. II Simposio sobre Igualdad y Distribución de la Renta y la Riqueza. Fundación Argentaria, Madrid, 1995.

COLLNS, E. y KLEIN, K. Equity and the NHS: self reported morbidity, access and primary care. BMJ 1980;281:1111-1115

CULYER, A.J. y WAGSTAFr, A. Equity and equality in health and health care. J Health Econ 1993;12:431-457.

CULYER, A.J. •Health, health expenditure and equity. University of York Discussion Paper, April 1991.

CULYER, A.J. Inequalities in health services is, in general, desirable, En GREEN, DJ (Ed.) Acceptable inequalities, The IEA Health Unit, London, 1988.

DHSS. Inequalities in beallh -Black Report- DHSS, London, 1980.

DIEZ, E., VILLALBI, J.R., BENAQUE, A. y NeBOT, M. Inequalities in matemal-child health: impact of an intervention, Gac Sanit 1995;9(49):224-31.

Doz, J.F., Gavagnach, M., Ros, E., Gracia, L.L., Romarguera, M., y Borrell, C. Breve revisión del debate sobre las desigualdades sociales en salud. Aten Primaria 1996;17(9):585-589

DuŔN, M.A. Desigualdad social y enfermedad. Tecnos, 1983.

Dworkin, R. . What is equality?. Philasopby and Public Affairs 1981;10:185-246 y 283345 .

ELOLA, F.J. Crisis y reforma de la asistencia sanitaria püblica en España (1983-1990). FISS, Madrid, 1991

ESCA. Enquesta de Salut de Catalunya. Sevei Català de la Salut, Generalitat de Catalunya, 1994.

FERNÁNDEZ DE LA HOZ, K. y LEÓN, D.A. Self-perceived health status and inequalities in the use of health services in Spain. Int J Epidemiol 1996;25(3):593-603.
Fox, J. Ed. Health Inequalities in European countries. Gower, 1989.

Fundación Argentaria. Las desigualdades en España. Sintesis estadística, Argentaria, Madrid, 1995.

Gallo, P., Serra-Prat, M. y Gravados, A. (en fase de edición). L'equitat en la provisió de serveis sanitaris a Catalunya. Informe de la Agència d'Avaluació de Tecnologia Mèdica, Servei Català de la Salut.

GaLlo, P. Salud, Sanidad y necesidades de la población. El Médico 1998;661:14-16.

GaLLO, P. PPolíticas de recursos y descentralización. En JuÁreZ et al., (Ed.) V Informe Sociológico sobre la Situación Social de España: sociedad para todos en el año 2000. Madrid 1994, Cap. 6.3:861-874.

Generalitat de Catalunya. Seminario sobre la distribución entre Comunidades Autónomas de los recursos públicos de sanidad. Quaderns de Treball 37, 1992.

GERDTHAM, U.G. Equity in health care utilisation: further tests based on the hurdle models and Swedish micro data. Health Economics 1997;6:330-319.

GONZÁLEZ, J. y REGIDOR, E. •Desigualdad en el uso de los servicios sanitarios. VIII Jornadas de Economia de la Salud, Ministerio de Sanidad y Consumo, 1988.

GREEN, D.J. Acceptable inequalities: Essays on the pursuit of equality in bealtb care. The IEA Health Unit, London, 1988.

Guluév, M.F. -Estructura social y Salud: análisis del acceso a la asistencia sanitaria y los niveles de salud a partir de la ENS. Estudios y Encuestas CIS N.22. Centro de Investigaciones Sociológicas, Madrid, 1990.

GuTtérRez, A. La sanidad pública como garantía de la equidad. Rev Adminis Sanit $1997 ; 1(2): 25-29$.

HASAN, J. Way-of-life, stress, and differences in morbidity between occupational classes. En Fox, J. (Ed.) Health Inequalities in European countries. Gower, 1989.

HOUSE, J.S. y KESSLER, R.C. •Age, socioeconomic status and health. The Milbank Quartely 1990;68(3): 383-411.

IBáNEZ-Fralle, A. y TEJEDOR, J. .Equidad en la demanda de hemodinámica y cirugía cardiaca en Castilla y León. Rev Calidad Asist 1995;10(1):7-12.

ILLSLEY, R. y SVENSSON, P.G. The bealth burden of social inequalities. WHO Regional Office for Europe, 1986.

JUDGE, K., MULUGAN, J.A., y BenZEVAL, M. .Income inequality and population health. Soc Sci Med 1998;46(4-6):567-57.

KuLlER, L.H. Editorial: The Use of Existing Databases in Mortidity and Mortality Studies. Am J Public Health 1995;85(9):1198-1200.

LaIRSON, D.R, Hindson, P. y HauquTtz, A. Equity of health care in Australia. Soc Sci Med 1995 41(4):475-482.

LaRDelu, P., Luna, J.D., Masa, J., LOPEZ, R., Delgado, M. y Gálvez, R. •Desigualdades en salud: la mortalidad perinatal e infantil en España. Gac Sanit 1993;34(7):21-26.

Latour-PÉrez, J., GuttérRez-Vicent, T., LOPEZ-Camps, V., Bonastre-Mora, J., GinerBOIX, J.S., RODRIGuez-SERRA, M. y ROSADO-BRETON, L. Differences in therapeutic effort because of the socioeconomic level of patients with AMI. Gac San 1995;9(40):5-10.

LE GRAND, J. -The distribution of health care revisited: A commentary on Wagstaff, van Doorslaer and Paci, and O'Donnell and Propper. Joumal of Health Economics. 1991;10:239-245.

LE GRAND, J. Three essays on Equity. STICERD, Welfare State Programme N.WSP/23, London, 1987a.

LE GRAND, J. Equity and Choice. Harper Collins, London, 1987b. 
LE GRAND, J. The strategy of equality. Redistribution and the social services. George Allen and Unwin, London, 1982.

LE GRAND, J. .The distribution of public expenditure: the case of health care. Economica 1978;45:125-142.

LeE, P., Moss, N. y Kriegre, N. Measuring Social Inequalities in Health. Public Health Reports. 1995;110(3):302-305.

LEMkOw, L. Socio-Economic Status differences in health. Soc Sci Med 1986;22(11):1257-1262.

Llorca, J., Prieto, M.D., Álvarez, C.F. y Delgado-Rodríguez, M. .Age differential mortality in Spain, 1900-1991. JEpidemiol Community Health 1998;52(4):259-61.

luengo, S., Lazaro, P., Madero, R., Alvira, F., Fitch, K., Azcona, B., Pérez, J.M. y CABALLERO, P. Equity in the access to mammography in Spain. Soc Sci Med 1996;43(8):1263-71

MACINTYRE, S. The role of health services in relation to inequalities in health in Europe. En Fox, J. (Ed.). Health Inequalities in European countries. Gower, 1989.

MACKENBACH, J.P., y KUNST, A.E. -Measuring the magnitude of socioeconomic inequalities in health: an overview of available measures illustratedwith two examples from Europe. Soc Sci Med 1997;44(6):757-771.

Manor, O., MatTHEwS, S. y POWER, C. .Comparing measures of health inequality. Soc Sci Med 1997;45(5):761-771.

Marín, N., Caba, A., Bernabé, O., Pérez-Tornero, E., Martínez, L., López, M., FornieLES, H. y DELGADO-RODRÍGUEZ, M. :Determinantes socioeconómicos y utilización de los servicios de urgencias. Med Clin 1997;108:726-729.

Marmot, M., RYFF, C.D., Bumpass, L.L., ShIPLEY, M., y MaRKS, N.F. Social inequalities in health: next questions and convering evidence. Soc Sci Med 1997;44(6):901-910.

MIGUEI, J.M. De. Salud y Sanidad. En JuÁREZ et al., (Ed.) V Informe Sociológico sobre la Situación Social de España: sociedad para todos en el año 2000. Madrid 1994 Cap.6:828-1087.

Miguel, J.M. De y Rodriguez, J.A. Salud y poder. CIS, Madrid, 1990

Miguel, J.M. De. Social inequalities related to health in Spain. En The bealth burden of social inequalities. WHO, 1986.

MiGuEL, J.M. De. La salud príblica del futuro, Ariel Sociologia, 1985.

Miguel, J.M. De. La sociedad enferma: las bases de la política sanitaria española, Madrid, 1979.

MiLs, A. et al., Health system decentralisation. Concepts, issues and country experience WHO, 1990

Ministerio Administraciones Públicas. Régimen de distribución de competencias entre Estado y Comunidades Autónomas: Sanidad. Secretaría de Estado Admin. Públicas, Madrid, 1992.

MOONEY, G.H. What does equity in health mean?. World Health Statistics Quarterly $1987 ; 4$.

MONROE, B.L. snequity, inequality and disproportionality: measuring deviations from a distributional standard. Working Paper N.113, 1992.

MOSSEY, J.M. y SHAPIRO, E. Self-rated health: a predictor of mortality among the elderly. Am J Pub Health 1982;72:800-808.

NAVARRO, A.E. Sistemes sanitaris integrats en els estats políticament descentralitzats. Quaderns de treball 351992
Navarro, V. y BeNaCH, J. -Informe de la comisión científica de estudio de las desigualdades sociales en salud en España. Rev Esp Salud Pub 1996;70(5-6):505-636.

NavarRo-Rubio, M.D., Jovell, A.J. y Schor, E.L. Socio-economic status and preventive health care use by children in Spain. Am J Prev Med 1995;11(4):256-62.

O'DONNEIL, O. y PropPER, C. .Equity and the distribution of UK NHS resources. Journal of Healtb Economics 1991a;10:1-19.

O'DONNELl, O. y PrOPPER, C. Equity and the distribution of UK NHS resources: a reply. Joumal of Health Economics 1991b;10:247-279.

PerelRA, J. .What does Equity in Health Mean?. J Soc Pol. 1993;22(1):19-48

POLAND, B., COBURN, D., ROBERTSON, A. y EAKIN, J. Wealth, equity and health care: a critique of a population health perspective on the determinants of health. Soc SCi Med 1998;46(7):785-798

PORTELA, E., BoliBar, B. y FerRUS, L. •Desigualdades en la frecuentación de los servicios de asistencia sanitaria. VII Jomadas de Economia de la Salut, 1988

PUFFER, F. •Access to primary health care: a comparison of the US and the UK. I SOC Pol 1985;15(3):293-313

PuYOL, A. slgualdad y sistema sanitario. Intervención Psicosocial 1997;6(3):301-309.

RAMIS, O., SOKOU, J. y SOKOU, K. Social health inequalities in South European countries: is it a different problem? Health Inequalities in European Countries. In Fox, J. (Ed.). Health Inequalities in European countries, Gower, 1989.

Regidor, E., NavarRo, P., Domfnguez, V. y Rodriguez, C. Inequalities in income and long-term diability in Spain: analysis of recent hypotheses using cross-sectional study based on individual data. BMJ 1997;315(7116):1130-5.

Regidor, E., De Mateo, S., Gutérrez-FisaC, J.L., Ferváivdez de la Hoz, K. y Rodríguez, C. Diferencias socioeconómicas en la utilización y accesibilidad de los servicios sanitarios en España. Med Clin 1996;107:285-288.

Ruco, A. Descentralización y reforma sanitaria en España (1976-1996). Tesis Doctoral Universidad Autónoma de Madrid. Departamento de Sociología, Ciencia Política y Antropologia Social, 1997.

RICO, A. LLa descentralización sanitaria en España: el camino recorrido y las tareas pendientes. Papeles de Economia Española 1998;76:49-66.

RODRIGUE2, J.A. y LEMKOw, L. .Health and social inequities in Spain. Soc Sci Med 1990;31(1):223-420.

Rodriguez, M., Calonge, S. y ReNE, J. .Spain. En VAN DOORSLAer et al., Equity in the finance and delivery of bealth care: an intermational perspective. Oxford University Press, Oxford, 1993.

ROEMER, M.I. National bealth systems of the world Vol.I and II. Oxford University Press, 1993

Rohlfs, I., Borrell, C., Plasencia, A. y Passarin, I. Social inequalities and realisation of opportunistic screening mammographies in Barcelona. J Epidemiol Community Heallb 1998;52(3):205-6.

RONDINEL, D.A. -Govemment decentralisation in comparative theory and practice in developing countries. Intermational Review of Administrative Sciences 1981;47-2:133145 .

ROSENBERG, M.W. y HANLON, N.T. •Access and utilization: a continuum of Health service environments. Soc Sci Med. 1996;43(6):975-983.

Rúz, T., Ronda, E., Alvardez-Dardet, C., Gil, V. y Belda, J. -Care according to patient gender in emergency services: different or unequal?., Gac Sanit 1995; $9(47): 76-83$. 
Sínchez-Fernández, F. Vázquez-Sánchez, M.A., Ramírez-Sánchez, M.J., Estebánez-CarVAJAL, F. y APARICIO-Benito, A. Programa de la mujer. Influencia de las desigualdades socioeconomicas en su cobertura. Rol 1995;18(197):19-25.

SCRIVENER, G. y LLOYD, D. .Two proxies for morbidity in the 1991 UK census: permanent sickness and limiting long tern illness. Joumal of Epidemiology and Community Health 1996;50: 223 .

Segurs, A., Fernández, E. -Mortality and low educational level. Med Clin 1998;110(5):177-9.

SHI, L. y LU, N. .Personal characteristics and self assessed health. Med Care 1997;35(10):1069-1078.

SIEGRIST, J. Social differentials in chronic disease: what can sociological knowledge offer to explain and possibly reduce them?. Soc Sci Med 1995;41(12):1603-1605.

SUCDEN, R. -Who Cares?. IEA Occasional Paper, N267, Institute for Economic Affairs, London, 1983.

TOWNSEND, P., SIMPSON, D. y TiBBS, N. Inequalities in health in the city of Bristol: a preliminary review of statistical evidence. International joumal of Health Services 1985;15(4): 637-663.

VÂGERO, D. eInequality in health - Some theoretical and empirical problems. Soc $S c i$ Med 1991;32(4):367-371.

VAlERO, C., VILLALBI, J.R., BORRELL, C. y NeBOT, M. Inequalities in health at birth: Barcelona, 1990-91., Aten Primaria 1996;17(3):215-9.
VAN DER MEER, J., VAN DEN BOS, J. y MACKENBACH, J.P. Socioeconomic differences in the utilization of health services in a Dutch population: the contribution of health status. Health Policy 1996;37:1-18.

VAN DOORSLAER, E., WAGSTAFF, A. y RUTTE: F. Equity in the finance and delivery of bealth care: an international perspective. Oxford University Press, 1993.

WADSWORTH, M.E.L. Health inequalities in the life course perspective. Soc Sci Med 1997;44(6):859-869.

WAGSTAFF, A. y VAN DOORSLAER, E. •Measuring inequalities in health in the presence of multiple-category morbidity indicators. Health Economics 1994;3:281-291.

WaGSTAF, A., VAN DOORSLaER, E. y PACI, P. .On the measurement of horizontal inequity in the delivery of health care. Joumal of Health Economics 1991a;10:169-205.

WaGSTAF, A., VAN DOORSLAER, E. y PACl, P. .Horizontal equity in the delivery of health care: a reply. Journal of Healtb Economics 1991b;10:251-256.

WAGSTAFF, A., PACI, P. y VAN DOORSLAER, E. On the measurement of inequalities in health. Soc Sci Med 1991c;33(5):545-557.

WHITEHEAD, M. Inequalities in bealth: The bealth divide. Penguin Books, 1992.

WHITELEGG, J. Inequalities in bealsh care: problems of access and provision. Strawbarnes, 1982.

ZiERIER, A. Socioeconomic Stattus and Health Care Services. Society Health 1996;3. 


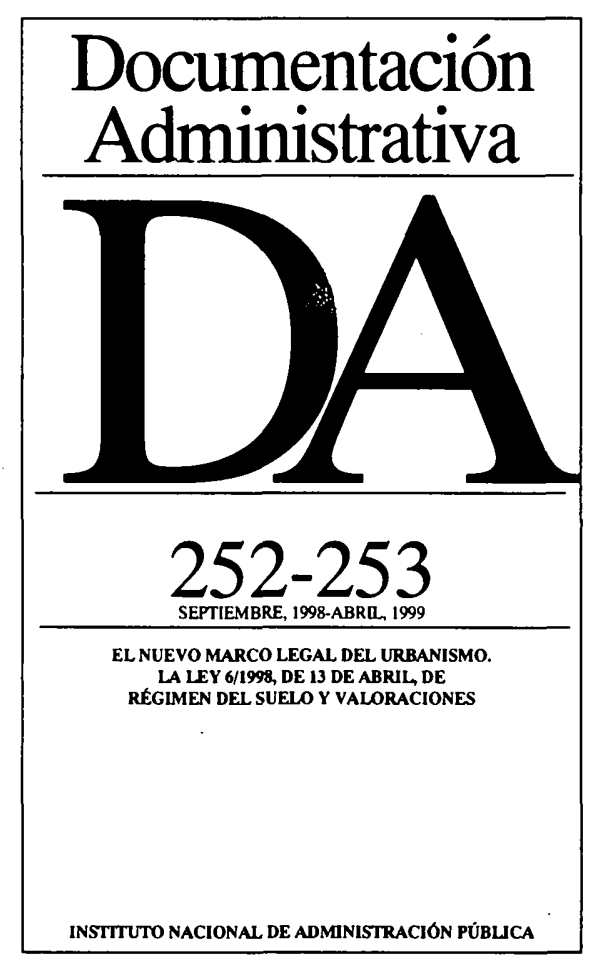

Publicación cuatrimestral del Instituto Nacional de Administración Pública, especializada en Derecho Administrativo y Organización y Gestión de las Administraciones Públicas.

Aparecida en 1958, la característica más destacada de su actual etapa es la dedicación monográfica de cada número a una cuestión específica, que se analiza desde diferentes puntos de vista por acreditadas firmas de España y otros países.

\section{CONSEJO DE REDACCIÓN}

Presidente: Enrique Álvarez Conde. Vocales: José Javier Abad Pérez, Óscar Alzaga Villamil, Manuel Aragón Reyes, Enrique Argullol Murgadas, Gaspar Ariño Ortiz, Miguel Beltrán Villalva, Andrés de Blas Guerrero, José Manuel Castells Arteche, Íñigo Cavero Lataillade, Rafael Gómez-Ferrer Morant, Francisco González Navarro, Jesús González Pérez, Luis Morell Ocaña, Sebastián Martín-Retortillo Baquer, José Ramón Parada Vázquez, Eduardo Roca Roca, Ángel Rodríguez García, Juan Alfonso Santamaría Pastor, Gumersindo Trujillo Fernández.

\section{Director}

Luciano Parejo Alfonso

Secretario

Jesús Prieto de Pedro

Edita:

MINISTERIO DE ADMINISTRACIONES PÚBLICAS

Instituto Nacional de Administración Pública

\section{Suscripciones y Administración:}

Instituto Nacional de Administración Pública

Publicaciones Periódicas

Atocha, 106. 28012 Madrid

Teléfono: 3493104

\section{Precios:}

